\title{
Evolution of unicellular eukaryotes and possible ways to multicellularity
}

\author{
I. Dovgal \\ Kovalevsky Institute of Marine Biological Research RAS, Sevastopol, Russia \\ e-mail: dovgal-1954@mail.ru
}

Key words: choanomonads, evolution, multicellularity, Metazoa

Motivation and Aim: Author's hypothesis on the possible continuity between the mechanisms of intercellular recognition in unicellular eukaryotes and the mechanisms of intercellular interactions in Metazoa is considered with the light of the latest data on the evolution, phylogeny and genomics of unicellular eukaryotes.

Results: E. Haeckel was first who proposed to combine both heterotrophic and autotrophic unicellular eukaryotes along with sponges and fungi in kingdom Protista, which was intermediate between kingdoms Planta and Animalia.

However, in accordance with more popular view the heterotrophic unicellulars were placed in kingdom Animalia as phylum Protozoa, while autotrophic in Planta. In turn, the relations within Protozoa were based on modes of locomotion, feeding and attended peculiarities of cell morphology.

In the subsequent, the assumption on the unicellular eukaryotes evolution have their bases in transmission electron microscopy (types of mitochondria crista) evidence.

However, the use of modern approaches has led to the rejection of morphological schemes, since the general evolutionary line of eukaryotic organisms has broken up into several molecular clusters, or supertaxa, that do not coincide with the morphological groups.

One of these supertaxa named Opisthoconta combines heterotrophic protists, including choanomonads, along with fungi and metazoans. As this takes place, the Choanomoada and Metazoa are sister groups, which confirms the views about the possible origin of Metazoa from the choanomonads, based on the similarity of the collar cell of choanomonads and sponge choanocyte morphology. Even if the Choanomonada are not the direct ancestors of sponges, the similarity possible reflects a general evolutionary trend, which allows tracing of possible ways of Metazoa origin.

Three general ways from unicellular eukaryotes to multicellular organization are recognized: complete or incomplete cell divisions within a common extracellular matrix, cellularization of a multinucleated cell and aggregations of cells attracted by chemical signals. The first of the ways, which gave rise to coloniality, seems most plausible. As this takes place, the cell differentiation into generating and transmitting signals in connection with increasing of colonies seems inevitable.

The mechanisms of regulation of protozoan ontogeny as well as mechanisms of intercellular interaction in these organisms may serve as a model for the formation of mechanisms of metazoans morphogenesis. This corroborated by information that in choanomonad Salpingoeca rosetta there are genes encode homologs of cell adhesion, neuropeptide, and glycosphingolipid metabolism, which previously found only in metazoans. In addition, the transcriptome analysis revealed that septins found in the species might regulate incomplete cytokinesis during colony development.

Acknowledgements: Supported by the grant of Ministry of Education of the Russian Federation (No. 14W03.31.0015). 